\title{
Soft X-ray solar flare cycles
}

\author{
Georgeta Mariş ${ }^{1}$, Miruna Daniela Popescu ${ }^{1,2}$ \\ and Marilena Mierla $\mathbf{a}^{1,3}$ \\ ${ }^{1}$ Astronomical Institute of the Romanian Academy Str. Cutitul de Argint 5, RO-040557 \\ Bucharest 28, Romania; e-mail: gmaris@aira.astro.ro \\ ${ }^{2}$ Armagh Observatory, College Hill, Armagh, BT61 9DG, N. Ireland; email: mdp@arm.ac.uk \\ ${ }^{3}$ Max-Planck Institut für Aeronomie, Katlenburg-Lindau, Germany; \\ email: mierla@linmpi.mpg.de
}

\begin{abstract}
We present a statistical investigation of soft X-ray (SXR) solar flare occurrence over the last three 11-yr solar cycles (SCs 21-23, corresponding to the period 1976-2003). We studied not only the monthly number of flares, but we also used an index that estimate the energy emitted by flares in the SXR 1-8 $\AA$ band $\left(Q_{x}\right)$ that we find it better defines their importance in the solar activity cycle. The medium and high importance SXR flares (M\&X class) seem to reflect the cycle importance given by the smoothed monthly Wolf numbers (W). SXR flare indices show a delay regarding the sunspot numbers for SC 21, while for SC 22 and 23 their time distribution is almost coincident with W. The level of energy emitted by SXR flares, quantified through the $Q_{x}$ index, is about the same for SCs 21-23 despite the difference between the W curves in the cycles. We also point out the SXR flare behaviour on the cycles descendant phases, with short but intense increases in their activity, that might give useful information about the activity level of the next cycle.
\end{abstract}

\section{Introduction}

Solar flares are the result of rapid conversion of large amounts of magnetic energy, stored in the corona, and dissipated through magnetic reconnections. They begun to be studied in the optical $H_{\alpha}$ emission line, being monitored from ground stations since 1936 . Since 1975 they are continuously registered also in soft X-ray (SXR), from space.

The number of different types of flares was often used as index in statistical studies of flare occurrence or their N-S asymmetry. This index reflects how frequently solar flares appear during one interval or in one solar hemisphere and gives little information about the associated energy release. Its main time variation is the 11-yr cycle. Other types of indices, which contain more information on the energy of these phenomena (importance indices), are also used to evaluate the energy released by flares (Kuklin 1976; Knoška 1985; Mariş 1987). Such indices are more useful in the analysis of flare spatial distribution, and reveal flare concentrations in active longitudes or in one of the solar hemispheres.

In this study we present a statistical investigation of SXR flares over the last three 11-yr solar cycles (SCs 21-23, the period 1976-2003) using two types of indices: a frequency index $-N_{x}$ - the total SXR flare number and an importance index $-Q_{x}$ (Mariş et al. 2002a) - that estimates the energy emitted by flares in the SXR 1-8 $\AA$ band.

\section{Data and methods}

We first analysed the time distribution of the SXR flares by the monthly values of $N_{x}$, during the interval Jan. 1976 - Dec. 2003 (Fig. 1, left panel). For estimating the flare 

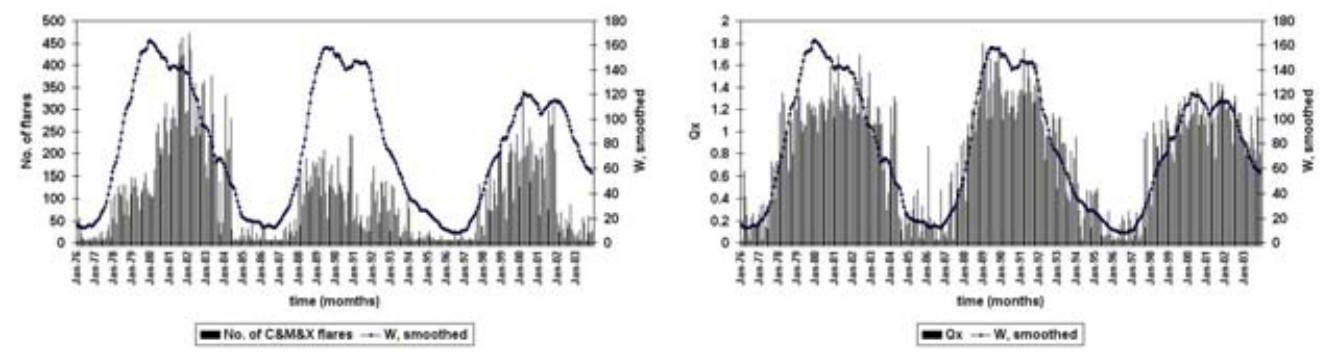

Figure 1. The monthly variation of the $N_{x}$ (left) and $Q_{x}$ (right) indices during the period Jan. 1976 - Dec. 2003. The W courve is superimposed.

energy release in the 1-8 $\AA$ band, we quantified them over a period of 24 hours calculating the index $Q_{x}$ defined by Mariş et al. (2002a), based on the spectral classification of solar flares according to the magnitude of the peak burst intensity within 1-8 $\AA$ measured from the Earth orbit. This evaluation is similar to that carried out on $H_{\alpha}$ flare energy release (Ataç \& Özgüç 1996; 2001). In Fig. 1 (right panel) we present the monthly variation of the $Q_{x}$ index. For better inferring the cycle characteristics of our indices, in Fig. 1 we also superimposed the smoothed monthly Wolf numbers (W).

\section{Analysis of the results}

The 11-yr cycle, generally defined through the sunspot relative number, is certainly the clearest periodicity observed also in $H_{\alpha}$ flare behaviour. However, the flare cycle has a longer maximum phase and the flares of small importance usually have their maximum before or coincident to the sunspots one, while the majority of more energetic flares occur later (Dinulescu \& Dinulescu 1991). On the descendant phase there are also some intervals with increased flare activity, greater than for sunspots, often placed near the minimum (Popescu et al. 2002). Most of those pulses from the descendant solar activity phases have geophysical effects on the terrestrial environment (Mariş et al. 2002b).

\subsection{Flare numbers}

The histogram representing the monthly values of $N_{x}$ (Fig. 1 left) shows specific characteristics during SCs 21-23. Therefore, for SC 21, $N_{x}$ is relatively small during 1976-77, growing up to about 100 and remaining under 150 for the next two and a half years. A new increase showed up after Jun. 1980 and the real maximum of $N_{x} \approx 450$ was registered in the second half of 1981, two yr after the W maximum. During 1982, the descendant phase began and two pulses were registered in 1983 and 1984, followed by a prolonged minimum of about three yr.

For SC $22, N_{x}$ showed a remarkable increase in 1988, a high activity during 1988-89 and a second maximum in the end of 1990, followed by an unexpected deep in 1991 . However, after 1991, the activity began the descendent phase with some pulses during 1993-95. The minimum phase lasted for about three yr.

During SC 23, $N_{x}$ increased after the end of 1997 until the year of maximum (2000). There was a high flare activity after Nov. 1999. The higest $N_{x}$ is recorded in Mar. 2000 (with 343 SXR flares), near the W maximum (Apr. 2000). The second maximum registered in Nov. 2001 (310 SXR flares), in phase with the second W maximum. It follows a sudden decrese in $N_{x}$, with small pulses of activity growths during 2002 and 2003.

SC 22 had the smallest number of SXR flares (only 8233, with 6030 of C spectral class and 2203 of M\&X class), even if it was comparable with SC 21 regarding the W 


$\begin{array}{cccc}\text { SC } & \text { phase } & \text { intervals } & Q_{x, m} \\ 21 & \text { T } & \text { Jun. 1976 - Aug. 1986 } & 0.81 \\ & \text { A } & \text { Jun. 1976 - Nov. 1979 } & 0.73 \\ & \text { D } & \text { Dec. 1979 - Aug. 1986 } & 0.85 \\ 22 & \text { T } & \text { Sept. 1986 - Sept. 1996 } & 0.81 \\ & \text { A } & \text { Sept. 1986 - Jun. 1989 } & 0.81 \\ & \text { D } & \text { Jul. 1989 - Sept. 1996 } & 0.81 \\ 23 & T^{*} & \text { May 1996 - Dec. 2003 } & 1.00 \\ 23 & \text { A } & \text { May 1996 - Mar. 2000 } & 0.66\end{array}$

Table 1. The mediated values of the $Q_{x}$ index for the total length $(\mathrm{T})$, the ascendant $(\mathrm{A})$ and descendant (D) phases of SCs 21-23.

maximum (in SC 21 there were 16877 SXR flares, with 14543 of C class and 2334 of M\&X class). SC 23 is not yet finished but it already had 9409 SXR flares, with 8417 of $\mathrm{C}$ class and only 992 of M\&X class.

\subsection{Flare importance indices}

In Fig. 1 (right) we give the monthly values of the $Q_{x}$ index during SCs 21-23. We summarize some general features as follows. There are large maximum phases of $Q_{x}$ index for all the considered SCs. The double maximum structure is present in the monthly variations of $Q_{x}$ for both SCs 22 and 23 as for W. The pulses of higher activity on the descendent phases and the oscillations during the minimum phases are present in all SCs.

The time variation of $Q_{x}$ generally follows the one of $\mathrm{W}$ in SCs 22 and 23. SC 21 presents a longer maximum phase (five yr, 1978-82) and a delayed maximum (placed in 1981-82) relative to W. SC 22 shows the same double maximum structure in 1989 and 1991, with the first peak higher, but close to the value of the second one. The growth in the activity of SC 23 is stepper, due to the high number of small importance flares.

When looking at the general layout of the monthly $Q_{x}$ index (Fig. 1, right), one can notice that their values are smaller for the current 11-yr cycle in comparison to the previous ones. That behaviour is in contradiction to the one displayed by $N_{x}$, which has a higher maximum in SC 23 than in SC 22. This trend was imposed by C-class flares, who release a small amount of energy.

For giving a quatitative evaluation of the SXR flare activity during the last three SCs, we calculated the mediated values, $Q_{x, m}$ by summing the monthly values of $Q_{x}$ in each considered interval, and then dividing it by the number of the months in the selected interval. The analysis was done for the total length (T) as well as for the ascendant (A) and descendant (D) phases of each cycle. As one can see in Table 1, the $Q_{x, m}$ values are practically the same for SCs 21 and 22. For SC 23 we do not have enough data for all estimations, and therefore we calculated $Q_{x, m}$ only until Dec. $2003\left(T^{*}\right)$ and ascendant phase. The last value is the smallest while the $T^{*}$ value is the highest among the considered SCs.

We also evaluated the maximum phases $(M)$ by their mediated values of $Q_{x, m}$ (Table 2 ) as well as the "pulses" of activity registered on the descendant phases, considering them as "small maxima". The $Q_{x, m}$ index in all maxima are higher than the values of other phases. The $Q_{x, m}$ values for both pulses of SC 21 are comparable to its value over the whole cycle. The same is valid also for the first pulse of SC 22, but the following pulses are lower. The pulses of SC 23 have $Q_{x, m}$ values comparable with that ones of SC 21 and remained practically constant. 


\begin{tabular}{cccc}
\multirow{2}{*}{ SC } & phase & intervals & $Q_{x, m}$ \\
21 & M1 & Feb. 1978 - Dec. 1982 & 1.22 \\
& P1 & May 1983 - Oct. 1983 & 1.00 \\
& P2 & Jan. 1984 - May 1984 & 1.10 \\
22 & M2 & Feb. 1988 - Feb. 1992 & 1.44 \\
& P3 & Feb. 1993 - Jun. 1993 & 1.00 \\
& P4 & Oct. 1993 - Mar. 1994 & 0.65 \\
& P5 & Jun. 1994 - Oct. 1994 & 0.35 \\
& P6 & Dec. 1994 - Mar. 1995 & 0.45 \\
23 & M3 & May 1999 - May 2002 & 1.17 \\
& P7 & Jul. 2002 - Jan. 2003 & 1.13 \\
& P8 & Mar. 2003 - Jul. 2003 & 0.92 \\
& P9 & Oct. 2003 - Dec. 2003 & 1.05
\end{tabular}

Table 2. The mediated values of the $Q_{x}$ index during the maximum phases (M1 - M3) and the "pulses" on the descendant phase for SCs 21 (P1, P2), 22 (P3-P6) and 23 (P7-P9).

\section{Conclusions}

It is difficult to define an index that encompasses all the information about the flare phenomenon, especially for evaluating the emitted energy. Attempting to asses through an index, as accurately as possible, the energy emitted by flares is useful for understanding the dynamical evolution of the solar magnetic field and of the solar cyclic activity, in general. The conclusions of our study can be summarised as following:

(a) SXR flares are better defined through importance indices, as regards the knowledge about the amount of energy released, then through frequency indices.

(b) The medium and high importance SXR flares (M\&X class) seem to reflect the cycle importance given by $\mathrm{W}$.

(c) SXR flare indices show a delay regarding the sunspot numbers for SC 21, while for SC 22 and 23 their time distribution is almost coincident with W.

(d) The level of energy emitted by SXR flares, quantified through the $Q_{x}$ index, is about the same for SCs 21-23 despite the difference between the W curves in the cycles.

(e) SXR flares have short but intense growths in activity on the SC descendant phase, when magnetic phenomena generated by both the old and the new magnetic dipol interact in the solar atmosphere.

(f) During the descendant and minimum phases of the SCs, by looking at the flare activity growths, we may infer useful information about the level of the next cycle.

\section{Acknowledgements}

G. M. would like to acknowledge the receivement of an IAU travel grant.

\section{References}

Ataç, T., Özgüç, A. 2001 Solar Phys., 198, 399.

Ataç, T., Özgüç, A. 1996 Solar Phys., 166, 201.

Dinulescu, S., Dinulescu, V. 1991 Rom. Astron. J., 1, 63.

Knoška, Š. 1985 Contr. Astrophys. Obs. Skalnaté Pleso, 13, 217.

Kuklin, G. V. 1976 in: V. Bumba and J. Kleczeck (eds.), Proc. UAI Symp. No. 71, Prague, 25-29 Aug. 1975, D. Reidel Publ. Comp., Dordrecht, 147.

Mariş, G., Popescu, M. D., Mierla, M. 2002a Rom. Astron. J. 12, 131.

Mariş, G., Popescu, M. D., Mierla, M. 2002b ESA SP-477, 451.

Mariş, G. 1987 in: Anuarul Astronomic, Ed. Acad. Rom., Bucharest, (in Romanian) p. 319.

Popescu, M. D., Mariş, G., Oncica, A., Mierla, M. 2002 ESA SP-506, 721. 